\title{
Distinctive dendritic cell subsets expressing factor XIlla, CD1a, CD1b and CD1c in mycosis fungoides and psoriasis
}

The papillary dermis of psoriasis and mycosis fungoides (MF) lesions is characterized by prominent collections of cells with dendritic morphology. Immunophenotypically distinct populations of cutaneous dendritic cells have been identified as CD1a+, FXIIIa-Langerhans cells (LC) and CD1a-, FXIIIa+ dermal dendritic cells (DDC). In this study, antibodies against the human CD1 cluster of antigens (i.e. CDla, CD1b and CD1c) and the DDC marker (FXIIIa) were used to further characterize the subsets of dendritic cells in normal skin as compared to neonatal foreskin, psoriasis and MF by both immunoperoxidase and double immunofluorescence techniques. Normal skin and foreskin epidermis and dermis contained few CDlb+ or CD1c+ cells along with normal numbers of CD1a+ LC and FXIIIa+ DDC. Both MF and psoriasis were characterized by $\mathrm{CD} l \mathrm{a}+$ cells in the epidermis and dermis. FXIIIa+ cells were greatly expanded in the upper dermis of MF lesions and to a lesser degree in psoriasis as has been previously described by our group. MF contained significantly increased epidermal and dermal CD1b+ (15.7/5 high power fields [HPF] and $59.7 / 5$ HPF respectively) and CD $1 \mathrm{c}+$ dendritic cells $(33.8 / 5$ $\mathrm{HPF}$ and $95.9 / 5 \mathrm{HPF}$ respectively), while in psoriasis these cells were not statistically different from normal skin. Double immunofluorescence studies revealed that some $(<25 \%)$ FXIIIa+ cells co-expressed CD1b and CD1c in MF $>$ psoriasis $>$ foreskin, while FXIIIa+ DDG never co-expressed CDla. Thus, in contrast to normal skin in which epidermal or dermal dendritic cells rarely express CD1b and CD1c antigens, these members of the CD1 family are upregulated on both LC and DDC in benign and malignant inflammatory states. Upregulation of CD1b and CD1c on MF epidermal and dermal dendritic cells, as compared to psoriasis, foreskin and normal skin, may be useful in the immunophenotypic recognition of $\mathrm{MF}$, as well as in helping to understand its immunobiology.

Fivenson DP, Nickoloff BJ. Distinctive dendritic cell subsets expressing factor XIIIa, CDla, CD 1b and CD1c in mycosis fungoides and psoriasis. J Cutan Pathol 1995: 22: 223-228. (C) Munksgaard 1995.

\section{David P. Fivenson', Brian J. Nickoloff ${ }^{2}$}

Departments of ${ }^{1}$ Dermatology, Henry Ford Hospital, ${ }^{2}$ Pathology, University of Michigan, Ann Arbor, USA
David P. Fivenson, Department of Dermatology, Henry Ford Hospital, 2799 West Grand Boulevard, Detroit, Michigan 48202, USA

Accepted November 14, 1994
A growing body of evidence is available regarding the role of dermal dendritic cells (DDC) (1). We have described prominent collections these cells (defined by factor XIIIa expression) in lesional skin from mycosis fungoides (MF), psoriasis and allergic contact dermatitis (2-5). Data from several laboratories have shown that DDC express a variety of surface antigens including: Thy-1, FXIIIa, CD14, 


\section{Fivenson \& Nickoloff}

EBM-11, CD36, CD11b, and CD11c, suggesting they are related to blood-derived monocytes and/or tissue macrophages $(6-10)$. Despite this immunophenotyping data, the exact relationship between the various epidermal and dermal dendritic cells remains to be elucidated $(1,2)$. Recently, protocols for purification of DDC have revealed that there are several subsets identifiable and that these cells are efficient antigen presenting cells which selectively activate Th1-type T cells (11, 12)

CDla, CD1b and CDlc antigens have been identified as markers for several of these cutaneous antigen presenting cells in benign lichenoid dermatoses such as lichen planus, allergic contact dermatitis and atopic dermatitis $(3,14)$. CD $1 \mathrm{c}$ has also been shown to be expressed by dermal endothelial cells (13). In this report, we have studied the distribution of antibodies which differentiate CD1 cluster of antigens (CDla vs CD1b vs CD1c) in relationship to FXIIIa in both the epidermal and dermis by immunostaining a series of specimens from normal adult skin, neonatal foreskin and untreated MF and psoriasis. We report that normal skin and foreskin contains rare $\mathrm{CD} 1 \mathrm{~b}+$ or $\mathrm{CD} 1 \mathrm{c}+$ dendritic cells similar to previous reports $(13,14)$. In contrast, epidermal and dermal CD1b+ and CD1c+ dendritic cells were regularly seen in both psoriasis and MF, FXIIIa+ DDC were found to be increased in both MF and psoriasis as we have previously shown. A prominent minority of these cells are shown to co-express CDIc and CDIb, but not CDla, in diseased skin only.

\section{Material and methods}

Patients - Punch biopsies from 16 patients with untreated MF (6 patch and 10 plaque-stage patients) and 9 patients with untreated plaque-type psoriasis were obtained after informed consent at the time of diagnostic biopsy procedure. Normal skin (non-sun exposed, $\mathrm{n}=7$ ) specimens were obtained from buttock skin of healthy volunteers. Neonatal foreskins $(n=11)$ were obtained within 4 $h$ of circumcision from the newborn nursery. All specimens were obtained under IRB approved protocols at Henry Ford Hospital and the University of Michigan Medical Center.

Immunohistochemistry - Immunoperoxidase staining was performed on air-dried, acetone-fixed, 4-micron thick cryostat sections of all specimens. Serial sections were exposed to primary antibodies for one hour. Antibodies were visualized using an avidin-biotin immunoperoxidase staining technique (Vectastain ABC Kit, Vector Labs Inc., Burlingame, CA) as previously described (4). The chromagen used was 3-amino, 9-ethylcarbazole (AEC-Sigma Co., St. Louis, MO). All sections were counterstained with $1 \%$ hematoxylin. An irrelevant, isotype-matched antibody (anti-murine IaP or antihuman FXIIIs) was included with each set of patient slides as a control.

The antibodies were diluted in phosphate buffered saline (PBS) with $0.5 \%$ bovine serum albumin (BSA-Sigma) and included: anti-human CDla (1:200), CD1b (1:25), CD1c (1:50) (Biodesign, Kennebunkport, ME), anti-factor XIIIa (1:400), anti-factor XIIIs (1:400) (Calbiochem, LaJolla, CA). Preliminary experiments on reactive lymph node and normal skin specimens were performed to determine the optimal dilution for each antibody. Anti-murine IaP-class II (1:50) was used as an isotype control (Accurate Chemicals, Inc., Westbury, New York).

Double immunofluorescence - Four-micron thick cryostat sections were fixed in cold acetone for ten minutes and air dried. Primary antibodies (diluted using PBS with $0.5 \%$ BSA) were mouse anti-human CD1a (1:100), CD1b (1:10), CDlc (1:25) and rabbit anti-human factor XIIIa (1:200). These were applied and incubated at room temperature for 45 min. The slides were washed for $10 \mathrm{~min}$ in PBS, and labeled secondary antibodies (goat antimouse-FITC) (Dako-1:40) and goat anti-rabbit-rhodamine (Calbiochem-1:20) were applied for 45 min. Controls included deletion of one or both primary antibodies, and/or one or both secondary antibodies.

All sections were examined by light microscopy, and the numbers of reactive cells for each monoclonal antibody recorded. Quantitation was limited to cells of dendritic morphology in MF, psoriasis, normal skin and foreskin in all staining studies and was performed by counting all cells reactive with the antibody in at least five consecutive 40x high power fields (HPF) of contiguous epidermis and dermis and expressed as mean $\pm \mathrm{SEM} / 5 \mathrm{HPF}$.

Statistical analysis - Pairwise comparisons (Wilcoxon tests) were made of the epidermal and dermal expression of CD la vs CD 1 b vs CDlc between MF (patch and plaque stage samples were evaluated separately and as one group), psoriasis, normal skin and neonatal foreskin. Descriptive statistics were performed using the Kruskal-Wallis test. Statistically significant differences between the 6 pairwise comparison tests were defined as $p$ $<0.0083$ after Bonferroni's adjustment for the comparison of multiple groups. 
Table 1. Differential distribution of CD1 antigens in normal skin, neonatal foreskin, psoriasis and mycosis fungoides

\begin{tabular}{|c|c|c|c|c|c|c|c|c|}
\hline & $\begin{array}{l}\text { MF-epidermis } \\
n=16\end{array}$ & $\begin{array}{l}\text { MF-dermis } \\
n=16\end{array}$ & $\begin{array}{l}\text { PS-epidermis } \\
n=9\end{array}$ & $\begin{array}{l}\text { PS-dermis } \\
n=9\end{array}$ & $\begin{array}{l}\text { NS-epidermis } \\
n=7\end{array}$ & $\begin{array}{l}\text { NS-dermis } \\
n=7\end{array}$ & $\begin{array}{l}\text { FS-epidermis } \\
n=11\end{array}$ & $\begin{array}{l}\text { FS-dermis } \\
n=11\end{array}$ \\
\hline CD1a & $59.4 \pm 9.5$ & $46.1 \pm 10.7 \#$ & $35.5 \pm 6.3$ & $9.9 \pm 2.5$ & $42.8 \pm 10.0$ & $4.3 \pm 1.6$ & $30.6 \pm 3.5$ & $0.8 \pm 0.50$ \\
\hline CD1b & $15.7 \pm 5.9^{\star *}$ & $59.7 \pm 14.3$ & $2.6 \pm 1.4$ & $6.4 \pm 2.0$ & $0.8 \pm 0.5$ & $4.5 \pm 2.1$ & 0 & $3.1 \pm 2.1$ \\
\hline CD1c & $33.8 \pm 9.6^{\star \star *}$ & $95.9 \pm 20.8 \#$ & $8.3 \pm 3.1$ & $21.6 \pm 4.7$ & $1.1 \pm 0.5$ & $8.4 \pm 4.0$ & $4.5 \pm 1.5$ & $26.4 \pm 5.1$ \\
\hline
\end{tabular}

MF = Mycosis fungoides, PS=Psoriasis, NS=Normal adult skin, FS=Neonatal foreskin

* $=$ mean \pm SEM of 5 -consecutive high power fields in the epidermis or dermis

${ }^{* *}=$ statistical significance increase in MF vs foreskin, $p<0.0083$ after Bonferroni's adjustment on the rejection level for the pairwise comparison of 6 Wilcoxon tests

${ }^{* * *}=$ statistical significance increase in MF vS NS and foreskin but not PS, $p<0.0083$ after Bonferroni's adjustment on the rejection level for the pairwise comparison of 6 Wilcoxon tests

\# = statistical significance increase in MF vS PS, NS and foreskin, $p<0.0083$ after Bonferroni's adjustment on the rejection level for the pairwise comparison of 6 Wilcoxon tests

\section{Results}

Immunoperoxidase staining-Immunostaining results for the expression of CDla, CDlb and CDlc antigens on dendritic cells in MF, psoriasis and normal skin are summarized in Table I. Normal skin was found to contain rare epidermal and dermal $\mathrm{CD} 1 \mathrm{~b}+$ or CD1c+ dendritic cells, while CD1a+ epidermal and dermal cells were found in proportions comparable to prior reports (14-16). Epidermal CD1b+ and CDlc+ cells were rarely seen in normal skin and foreskin specimens. Those observed were found in a suprabasal location and had prominent dendritic processes. Foreskins tended to have greater numbers of CD1c+cells than normal skin, but these numbers were not statistically significant (Fig. 1a).

CD1b+ dendritic cells were significantly increased in the epidermis and dermis of MF lesions only in comparison to foreskin $(p<0.083)$ but were marginally increased in comparison to psoriasis and normal skin $(p=0.021$ and $p=0.023$ respectively) (see Table I and Fig. 1b). CDlct dermal dendritic cells were statistically increased $(\mathrm{p}<0.0083)$ in MF as compared to psoriasis, normal skin and/or foreskin specimens (Fig. 1c). CD1c+ epidermal cells were also statistically increased in MF as compared to normal skin and foreskin $(p<0.0083)$ but only marginally increased in comparison to psoriasis $(p=0.02)$ (see Table I and Fig. 1d). When analyzed separately, there were no appreciable differences in the mean numbers of CD1b+ or CD1c+ dendritic cells between patch and plaque stage MF specimens, therefore all statistical results represent the composite findings from the entire group of $16 \mathrm{MF}$ patients.

LC were regularly identified in all specimens as CD1at dendritic cells in a suprabasal epidermal location, but were not significantly increased in MF as compared to normal skin, as previously observed $(15,16)$. CDIb+ and CDlc+ dendritic cells were also seen in both compartments in psoriasis (Fig. 1d) but were not statistically increased in comparison to foreskin or normal skin numbers of cells. It could not be determined if CD1b+ or CD1c+ epidermal cells appreciably co-localized with GDlat cells in serial sections. CD lat dendritic cells in the dermis of MF were significantly increased over those in psoriasis, normal skin and foreskin dermis $(\mathrm{p}<0.0083$, see Table I). CDla+ epidermal and dermal cells in psoriasis were not significantly different from normal skin.

As compared to the numbers of FXIIIa+ dermal dendritic cells observed in normal skin (20-40/ 5 HPF) or neonatal foreskin (30-60/5 HPF), FXIIIa+ dendritic cells were greatly expanded in the upper dermis of MF lesions (100-300/5 high power fields[HPF]) and in psoriasis (50-70/5 HPF). The IaP and FXIIIs isotype controls were negative in all experiments (results not shown). No FXIIlat cells were found in the epidermis of any specimen.

Double immunofluorescence staining - Double IF studies were performed on all patient specimens. $10-25 \%$ of the FXIIIa+ DDC were found to $\mathrm{CO}^{-}$ express $\mathrm{CD} 1 \mathrm{c}+$ in both MF and psoriasis, while few $(<10 \%)$ FXIIIa+ DDC co-expressed CD1b in both $\mathrm{MF}$ and psoriasis. Precise quantification was not possible due to the extensive dendritic arborizations present throughout the inflammatory lesions of MF and psoriasis (Fig. 2a, 2b). No FXIIIa+ cells were found to co-express CDla in normal skin, foreskin, MF or psoriasis.

\section{Discussion}

This study demonstrates that dendritic cell populations in both epidermal and dermal skin compartments can express of CD1b and CDlc antigens in inflammatory states. We have shown that epidermal CD1b+ and CD1c+ dendritic cells were rarely found in normal adult skin but were regularly recognized in MF and psoriasis. CDla+ LC and FX- 


\section{Fivenson \& Nickoloff}
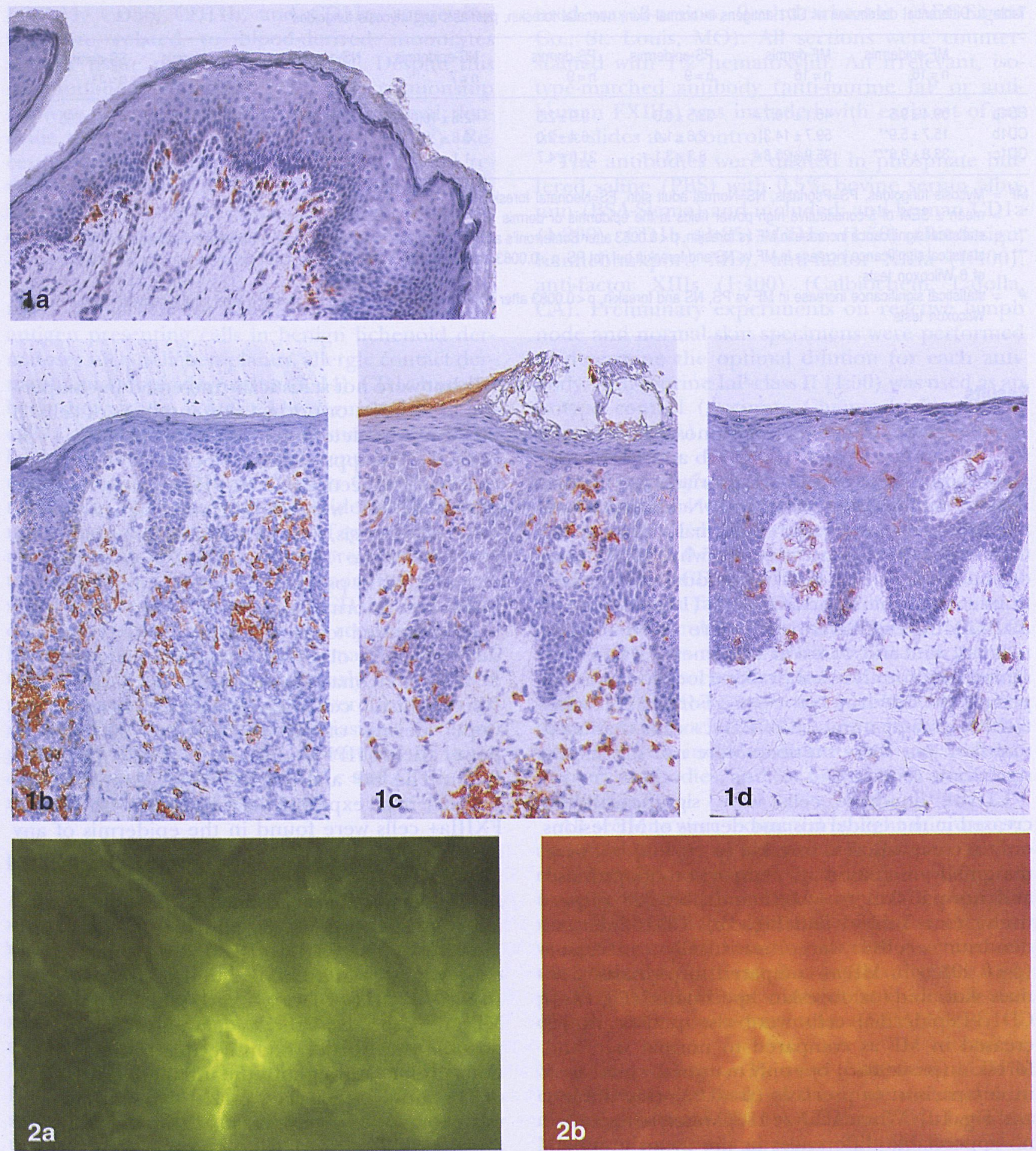

Fig. 1. Immunoperoxidase staining of $\mathrm{CDIb}+$ and $\mathrm{CD} 1 \mathrm{c}+$ dendritic cells in foreskin epidermis and dermis (Fig. 1a-CDlc, $\times 50)$, plaque stage mycosis fungoides (Fig. 1b-CD1b, $\times 50$; Fig, 1c-CD1c, $\times 50$ ) dendritic cells and in lesional psoriasis (Fig. 1d-CD1c, $\times 50$ ). There are several prominently stained dendritic cells in the dermis as well as in the epidermis in both Figs. 1b and 1c. Mycosis fungoides was found to have the greatest increase in CD1c+epidermal and dermal dendritic cells compared to normal skin (see text).

Fig. 2. Double immunofluoresence labelling of dermal dendritic cells in plaque stage mycosis fungoides. Fig. 2a- A CD1c+dendritic cell is stained green with the fluoresceinated secondary antibody (100). Fig. 2b-The same cell is also FXIIIa+ and stains red with the rhodamine labeled secondary antibody (100X). Note the prominent dendritic processes are present in both panels demonstrating co-expression by the identical cell. 
IIIa+ DDC were also found to be increased in MF, as previously reported $(4,6)$. These increases coincided with statistically increased numbers of $\mathrm{CD} 1 \mathrm{~b}+$ and $\mathrm{CD} 1 \mathrm{c}+$ dendritic cells in both the epidermis and dermis as compared to normal skin and/or neonatal foreskin.

A subset of FXIIIa+ dermal dendritic cells in all cases of MF, psoriasis and foreskin were found to co-express CD1c+ and to a lesser degree CD1b+, yet never expressed CDla. CD1b+ and CD1c+ FXIIIa+ DDC were rarely seen beyond their routine perivascular location in normal adult skin. Dermal co-expression of FXIIIa and CD1 cluster antigens beyond CDla (i.e. CDlb and/or CDlc) by dendritic cells in psoriasis and MF suggests that there are distinct subsets of dermal dendritic cells that become apparent under certain inflammatory and neoplastic conditions (14). Unique immunophenotypes appear to exist in epidermal vs dermal compartments, as co-expression of CDla and FXIIIa was not shown. Thus, although similar in morphology, DDC remain as distinct from LC. Recently, subpopulations of dermal dendritic cells have been isolated from psoriasis and normal skin and a CD14+, FXIIIa+, CD1a+ subset was found in vitro $(11,12)$.

These data give no direct insights into the functional properties of DDC vs. LC. However, CD1 antigens have been shown to resemble HLA- Class Ib molecules which are selectively involved in antigen presentation to CD8+ cells (15). Physiologic upregulation of these CD1 antigens in fetal and neonatal skin could help in generation of selftolerant clones of CD8+ cells. Aberrant CD1b or CD1c expression by dendritic cells in MF and psoriasis might be a mechanism through which autoreactive signals are generated which allow disease progression or a mechanism through which cytotoxic $\mathrm{T}$ cell are generated locally. Taylor et al. recently reported CD1b and CD36 "aberrantly" expressed by epidermal LC as well as CD1a+, CD1b+, CD36+, HLA-DR+ dermal cells in association with increased autoreactivity in atopic dermatitis (9). CD1a-, CD36+ macrophages in man and Thy-1+ dendritic epidermal $\mathrm{T}$ cells in mice have also been reported to possess this type of suppressor-inducer function in vitro $(9,17-20)$.

While the function of FXIIIa+ DDC is unknown , this study demonstrates that certain subsets of epidermal and dermal dendritic cells are interrelated immunophenotypically. CD1b and CD1c expression by FXIIIa+ DDC, while rare in normal skin, is shown to be more common in inflammatory skin conditions. We speculate that, in T cell-mediated skin disease (e.g. psoriasis and MF), some unknown stimulus is functioning to regulate CD1b and CD1c expression on both DDC and LC. The mediators which regulate dendritic cell expression of CD1 cluster antigens are currently under investigation. The upregulation of $\mathrm{CD} 1 \mathrm{~b}$ and $\mathrm{CD} 1 \mathrm{c}$ expression in all lesional MF specimens on epidermal and dermal dendritic cells demonstrated here as compared to psoriasis, foreskin and normal skin, may be useful in the immunophenotypic recognition of MF, as well as in helping to understand its immunobiology.

\section{Acknowledgements}

0T. Ames, P. Christopherson and J. Corbett provided important immunohistochemical staining/. processing of tissue specimens. The authors thank John T. Headington, for critical review of this manuscript. This work was presented in part at the combined Society of Investigative Dermatology and American Federation of Clinical Research Meetings, Baltimore, Maryland, May 1992. Clin Res 40:499A, 1992, and was supported in part by NIH grants AR31857, AR40065, AR01823, AR40488 (BJN).

\section{References}

1. Headington JT. The dermal dendrocyte. In: Advances in dermatology, Callen JP, Dahl MV, Golitz LE, et al. eds, Chicago: Yearbook Publishers, Inc. 1986: 159.

2. Cerio R, Griffiths CEM, Cooper KD, Nickoloff BJ Headington JT. Characterization of factor XIIIa positive dermal dendritic cells in normal and inflamed skin. Br J Dermatol 1989: 121: 421.

3. Fivenson DP, Rheins LA, Nordlund JJ, Krull EA. Thy-1 expression and $\mathrm{T}$ cell receptor type in mycosis fungoides and benign dermatoses. J Natl Cancer Inst 1991: 83: 1088.

4. Fivenson, DP, Douglass MC, Nickoloff BJ. Cutaneous expression of Thy-1 in mycosis fungoides. Am J Pathol 1992 141: 1373.

5. Griffiths CEM, Nickoloff BJ. Keratinocyte intercellular adhesion molecule-1 (ICAM-1) expression precedes dermal $\mathrm{T}$ lymphocytic infiltration in allergic contact dermatitis (Rhus dermatitis). Am J Pathol 1989: 135: 1045.

6. Cerio R, Spaull JR, Wilson Jones E. Identification of factor XIIIa in cutaneous tissue. Histopathol 1988: 13: 362.

7. Cerio R, Spaull J, Oliver GF, Wilson Jones E. A study of factor XIIIa and Mac 387 immunolabelling in normal and pathological skin. Am J Dermatopathol 1990: 12: 221.

8. Weber-Mattheisen K, Sterry W. Organization of the monocyte/macrophage system of normal human skin. J Invest Dermatol 1990: 95: 83.

9. Hansen ER, Baadsgaard O, Lisby S, Cooper KD, Thomsen K, Vejlsgaard GL. Cutaneous T-cell lymphoma lesional epidermal cells activate autologous CD8 + T lymphocytes: involvement of both CD1+, OKM5+ and CD1+, OKM5- antigen-presenting cells. J Invest Dermatol 1990: 94: 485.

10. Walsh LJ, Goerdt S, Pober JS, Sueki H, Murphy GF. MS-1 sinusoidal endothelial antigen is expressed by Factor XIIIa+, HLA-DR+ dermal perivascular dendritic cells. Lab Invest 1991: 65: 732. 


\section{Fivenson \& Nickoloff}

11. Nestle FO, Zheng XG, Thompson CB, Turka LA, Nickoloff BJ. Characterization of dermal dendritic cells obtained from normal human skin reveals phenotypic and functionally distinctive subsets. J Immunol, 1993: 151: 6535: 6545.

12. Nestle FO, Turka LA, Nickoloff BJ. Characterization of dermal dendritic cells in psoriasis. Autostimulation of $\mathrm{T}$ lymphocytes and induction of Th1 type cytokines. J Clin Invest 1994: 94: 202.

13. Furue M, Nindl M, Kawabe K, Ishibashi Y, Sagawa K. Epitopes of CDla, CD1b, and CD1c antigens are differentially mapped on Langerhans cells, dermal dendritic cells, keratinocytes, and basement memebrane zone in human skin. J Am Acad Dermatol 1992: 27: 419-426.

14. Taylor RS, Baadsgaard O, Hammerberg C, Cooper KD. Hyperstimulatory CDla+/CD1b+/CD36+ Langerhans cells are responsible for increased autologous T lymphocyte reactivity to lesional epidermal cells of patients with atopic dermatitis. J Immunol 1991: 147: 3794.

15. Boumsell L, Knowles R. Summary of the T1 workshop. in "Leukocyte Typing III". AJ McMichael, ed. Oxford: Oxford Univ Press, 1987: 71.
16. Fujita M, Horiguchi Y, Miyachi Y, Furukawa F, KashiharaSawami M, Imamura S. A subpopulation of Langerhans cells (CD1a+Lag-) increased in the dermis of plaque lesions of mycosis fungoides. J Am Acad Dermatol 1991: 25: 491.

17. Lisby S, Baadsgaard O, Cooper KD, Vejlsgaard GL. Decreased number and function of antigen-presenting cells in the skin following application of irritant agents: relevance for skin cancer? J Invest Dermatol 1989: 92: 842.

18. Amornsiripanitch S, Barnes LM, Nordlund JJ, Trinkle LS, Rheins LA. Immune studies in the depigmenting C57BL/ Ler-vit/vit mice: An apparent isolated loss of contact hypersensitivity. J. Immunol 1988: 140: 3438.

19. Tigelaar RE, Lewis JM, Bergstresser PR. TCR $\gamma / \delta+$ dendritic epidermal T cells as constituents of skin-associated lymphoid tissue. J Invest Dermatol 1990: 94: 58s.

20. Chambers DA. The Thy-1 epidermal cell: perspective and prospective. Br J Dermatol 1985: 113 (suppl 28): 24. 
This document is a scanned copy of a printed document. No warranty is given about the accuracy of the copy. Users should refer to the original published version of the material. 